\title{
The Linear Formulation of Thermal Unit Commitment Problem with Uncertainties through a Computational Mixed Integer
}

\author{
Mian Khuram Ahsan, Tianhong Pan*, Zhengming Li \\ School of Electrical Information \& Engineering, Jiangsu University, Zhenjiang, China \\ Email: *thpan@ujs.edu.cn
}

How to cite this paper: Ahsan, M.K., Pan, T.H. and Li, Z.M. (2018) The Linear Formulation of Thermal Unit Commitment Problem with Uncertainties through a Computational Mixed Integer. Journal of Power and Energy Engineering, 6, 1-15. https://doi.org/10.4236/jpee.2018.66001

Received: May 6, 2018

Accepted: June 17, 2018

Published: June 20, 2018

Copyright ( $\odot 2018$ by authors and Scientific Research Publishing Inc. This work is licensed under the Creative Commons Attribution International License (CC BY 4.0).

http://creativecommons.org/licenses/by/4.0/

\begin{abstract}
The solar and wind renewable energy is developing very rapidly to fulfill the energy gap. This specific increasing share of renewable energy is a reaction to the ecological trepidations to conciliate economics with security due to the new challenges in power system supply. In solar and wind renewable energy, the only partially predictable is the output with very low controllability which creates unit commitment problems in thermal units. In this research paper, a different linear formulation via mixed integer is presented that only requires "binary variables" and restraints concerning earlier stated models. The framework of this model allows precisely the costs of time-dependent startup \& intertemporal limitations, for example, minimum up \& down times and a ramping limit. To solve the unit commitment problem efficiently, a commercially available linear programming of mixed-integer is applied for sizeable practical scale. The results of the simulation are shown in conclusions.
\end{abstract}

\section{Keywords}

Thermal Generating Unit, MILP (Mixed Integer Linear Programming), Unit Commitment

\section{Introduction}

\subsection{Background and Motivation}

The new era of technology created a competitive environment in power system which needs perfect and useful tools to support the resources scheduling decisions. In a centralized power system, the unit commitment problem has been solved traditionally by determination of time when to start up or shut down the thermal generating units to fulfill the demands while dispatching online. Keep- 
ing in view of the spinning reserve necessities by satiating all generation constraints such as ramping limits, production and up \& down minimum times, this operation is made over an explicit short-term time duration, to minimize inclusive process cost [1].

The independent system operator (ISO) solves the generating scheduling problems in present electricity market [2], which is similar as a problem of unit commitment in non-viable centralized Power System which is sponsored by Standard Market Design FERC's [3]. The critical intangible dissimilarity in both problems is instead of minimizing operation costs; the independent system operator maximizes social welfare measure which is a function of bids and offers market participant. Consequently, the solution of the old-fashioned centralized unit commitment problem for the economic power industry is relevant.

\subsection{Literature Review}

In the field of potential saving for several decades the combinatorial of mixed-integer on a large scale and the nonlinear programming problem is a vigorous topic for researchers. As a significance numerous elucidations methods have been offered such as dynamic programming [4] [5] [6], heuristics [7] [8] [9] [10], MILP (Mixed-Integer Linear Programming) [11] [12] [13] [14] [15], simulated annealing [16] [17], Lagrangian Relaxation [12]-[18]. In [19] an extensive recent literature review on unit commitment can also be seen.

The Dynamic programming is a way to solve problems of different variety of sizes, which can be easily changed to the model features of explicit utilities. The disadvantage of dynamic programming is that, it needs to limit the commitments measured at any time period and the suboptimal treatment of constraints (minimum up/down time) along with startup costs (time dependent). The heuristic algorithm is used when exact solutions are ineludibly computationally costly but imprecise solutions are adequate and produce solution independently. The hindrance of heuristic algorithm produces near optimal outcomes very rapidly. So there is always doubt of correctness. The Lagrangian Relaxation is utilized in manufacture UC programs more than Dynamic. The sub-optimality moves to zero when the number of units rises. One of its characteristic is that, it easily accommodates to the model characteristic of specific utilities. The key drawback of lagrangain relaxation is its intrinsic sub-optimality. The simulated annealing is the analogy among annealing procedure and optimization problem. Its strong feature is being autonomous to the preliminary solution and mathematical intricacy.

The unique advantages of mixed integer linear programming are as follows: 1) the parameter and structural can be achieved at the same time. 2) The discrete and logical constraints can be fingered plainly with binary numbers. 3) With mathematical tools a general systematic framework can be expressed for different problems. 4) It can deal with binary variables (linear) and separable from continuous variable (nonlinear). 5) Its efficient representation leads to super- 
structure which can deal with problems of any size in convenient time. 6) The efficient optimization can also exploit the structure of problems. In a finite number of steps, the MILP warranties the convergence to the optimal solution [11] by providing an accurate and flexible modeling framework. Furthermore, the optimal solution is available in the proximity while searching the problem tree. The branch-and-cut algorithm, which is an efficient mixed-integer linear software, has been proposed and currently available with a large scale capability and optimized as a commercial solver. That's why MILP based approaches received a great deal of attention as a consequence.

The MILP was applied in [11] to resolve the unit commitment problem with a formulation is composed of a set of three binary variables. These variables have modeled the startup \& shutdown, the ON \& OFF states for every unit and the subject period. In [1] the mixed-integer linear formulation was modeled with an extended form to face the self-scheduling problem in the electricity market by a single generating unit. At the expense of binary variables increasing numbers, many cost and constraints are included such as time-dependent startup cost, non-convex production cost, the intertemporal constraints (ramping limits) minimum up and down times.

In the realistic scenario, the power system consists of several generators, and the model in [12] and [11] needs binary variables in large numbers. Therefore, the ensuing MILP problems maybe intensively calculative for branch-and-cut algorithm implementation [12] [13] with its current computing capabilities.

The linear expression, which requires a single binary variable, was formulated in [20] with start-up costs and minimum up \& down times. Furthermore, on the spinning reserve constraints, the influence of ramping limits is not considered, and also shutdown cost is not included either.

A linear formulation is presented in this paper for the thermal unit commitment with an alternative mixed-integer which is symbolized by MILP-UC which needs a binary variable with a single set of one per unit as per period. In contrast to different MILP previous methods [12] [13], the binary variables with a lower number of MILP-UC produce a decrease when branch-and-cut algorithm search tree used for some nodes. Thus the solver uses less computational time to solve the realistic case. Furthermore, the MILP-UC precisely mockups the thermal unit commitment states, which improves the modeling capability in intertemporal constraints and time depended on startup costs [20].

The proposed model is also capable of solving the problems of scheduling which arises in markets for electricity furthermore, Market clearing processes (solved by ISO's), self-scheduling problems (solved by generating companies) to originate bidding tactics which are used to analyze the market participant's behavior by using market simulators. Hence this MILP-UC can also benefit the market agents.

\subsection{Contribution}

This paper contributes to a new formulation, of MILP-UC which needs less bi- 
nary variables and constraints to model the thermal unit commitment problem which can accurately reduce the burden of computational on existing MILP approaches. Furthermore, the scientific experience is presented by resolving a real application.

\subsection{Paper Organization}

The proposed MILP-UC detailed description is provided in this section. Furthermore, it also includes the intertemporal and physical constraints as a precise model for power generators. While in section III, the numerical outcomes are discussed and presented. In IV Section, the appropriate and relevant conclusion is presented. Lastly, in the appendix, the statistics cast-off for the simulation is given.

\section{Unit Commitment}

\subsection{Modeling Assumptions}

The assumptions playing in the proposed model are out listed below in numerical order for the purpose of clarity.

1) A Pool-Based energy-only power wholesale marketplace is considered where a market agent Clears the market one time a day with day-ahead and hourly-horizon. The market operator seeks to minimalize the total generation cost considering the generation price generation limits submitted by the generation unit, as well as the demand and reserve existing in this market. The market clearing outcomes are hourly unit state, production, start-up and shunt down cost.

2) Reserve in the market is set to be $10 \%$ of overall system demand, which reflects the GB market. However, the reserve can be adjusted accordingly, which will be considered in future work.

\subsection{UC Optimization Model}

In the model, the most critical task is to minimize and control the Total Operation Cost (TOC). The TOC is directly proportional to three main components the overall cost of production and the cost of startup and shutdown which can be formulated as [1].

$$
\begin{gathered}
\min \sum_{j, k}\left(c p_{j}^{k}+c d_{j}^{k}+c u_{j}^{k}\right) \\
V=\left\{p_{j}^{k}, v_{j}^{k}, c u_{j}^{t}, c d_{j}^{k}\right\}
\end{gathered}
$$

In scheduling Problems usually, the quadratic production cost function is used which can be written as (3). As presented in Figure 1 the cost function in (3) can be precisely approximated by a piecewise block. Moreover, power output is referred as a quadratic function of Production cost, while before initiating the start-up cost is modeled for the offline time as exponential function [1].

$$
c p_{j}^{k}=A_{j}^{k}+B_{j}^{k}\left(p_{j}^{k}-P_{\min j}\right), \forall j, k
$$




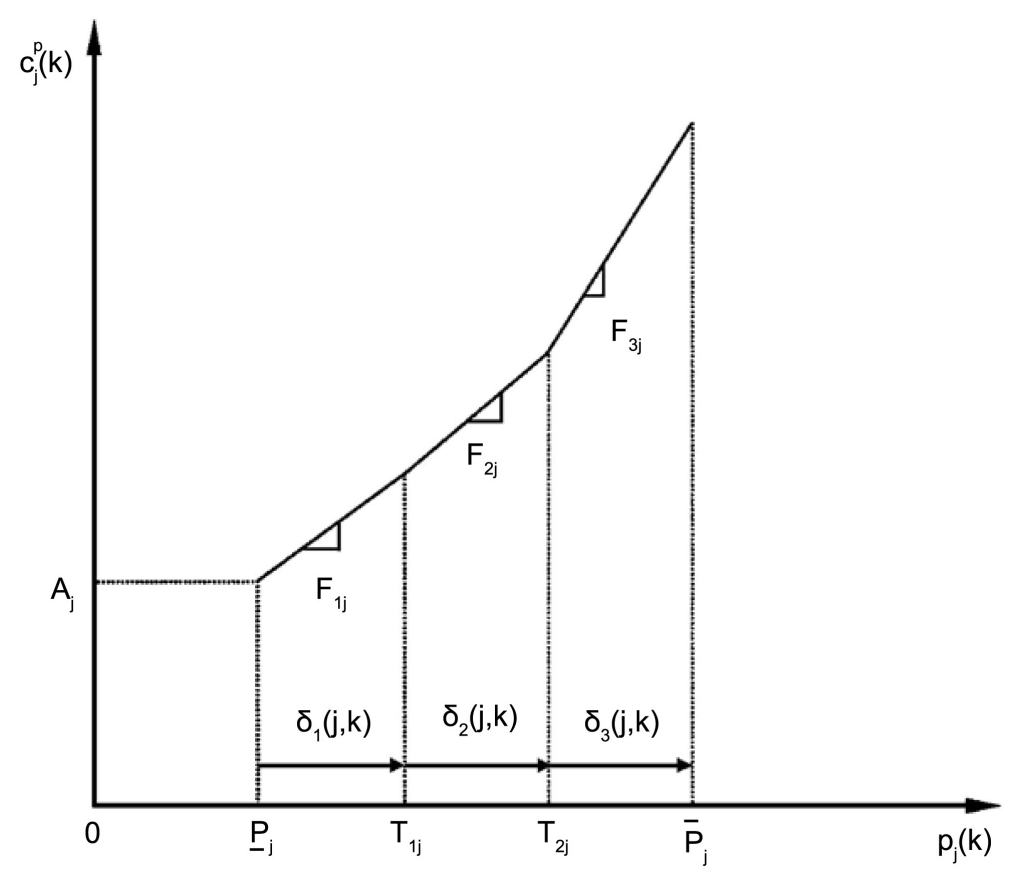

Figure 1. Piecewise linear production cost.

The stair wise start-up cost is proposed with the help of a mixed integer linear formulation. The stair wise function asymptotically approximates the discrete start-up cost which is a more accurate due number of intervals as increased. As the time span, the start-up cost is decentralized in hourly periods, so it can be written as a discrete function of start-up cost as shown in Equation (4).

$$
A_{j}^{k}=a_{j} v_{j}^{k}+b_{j} P_{\min j}+c_{j} P_{\min j}^{2}, \forall j, k
$$

In [10] and [11] with shutdown state, extra binary variables are associated. In the scenario of wastage of fuel [1] if unit $j$ is kept offline then the shutdown cost incurred can be set as constant. As shown in Equation (5).

$$
B_{j}^{k}=b_{j}+2 c_{j} P_{\min j}, \forall j, k
$$

With the span of time for every generating unit set of constraints are formulated as thermal constraints. The Power security constraints as shown in (6) provide spinning reserve margins, where the reserve in the market is set to be $10 \%$ of overall system demand, which has been assumed in Section III-A.

$$
\begin{gathered}
c u_{j}^{t} \geq C U_{j}^{t}\left(v_{j}^{k}-\sum_{n=1}^{t} v_{j}^{k-n}\right), \forall j, k, t \in\{1, \cdots, k-1\} \\
c d_{j}^{k} \geq C D_{j}\left(v_{j}^{k-1}-v_{j}^{k}\right), \forall j, k \in\{1, \cdots, T\}
\end{gathered}
$$

Power Security: constraints (8) provide spinning reserve margins, where the reserve in the market is set to be $10 \%$ of overall system demand, which has been assumed in Section III-A.

$$
\sum_{j \in J} p_{j}^{k} \geq D^{K}+R^{K}, \forall k
$$

The (9) constraints confine the minimum power output of the generation and 
with an all-out existing power output of unit-j in $k$ period, $\bar{p}_{j}$, It is a non-negative bounded variables of the above unit capacity (10).

$$
\underline{P}_{j} v_{j}^{k} \leq p_{j}^{k} \leq \bar{p}_{j} v_{j}^{k}, \forall j, k
$$

Note here that If in the period of $k$, the unit $j$ is offline then $v_{j}^{k}=0$, so both $p_{j}^{k}$ and $\bar{p}_{j}$ will be equal to 0 .

$$
0 \leq \bar{p}_{j} \leq \bar{P}_{j} v_{j}^{k}, \forall j, k
$$

In (11) the rates of ramp-up and start-up and in (13) the shutdown ramp rates both constrained the $\bar{p}_{j}^{k}$ while (12) represents the constraints in which ramp-down limits are enforced on the power output. In [21] the spinning reserve contribution of each unit in each period is precisely modeled which is extended by formulation (9)-(13), which can compute easily $\bar{p}_{j}^{k}$ and $p_{j}^{k}$ difference. It's important to note here that in (8) spinning reserve constraints which includes variables $p_{j}^{k}$ to fulfill the ramping-limitations and yielding the actual operation of generating units by an accurate representation.

$$
\begin{gathered}
\bar{p}_{j}^{k} \leq p_{j}^{k-1}+R U_{j} v_{j}^{k-1}+S U_{j}\left(v_{j}^{k}-v_{j}^{k-1}\right)-\bar{P}_{j}\left(1-v_{j}^{k}\right), \forall j, k \in\{2, \cdots, T\} \\
p_{j}^{k} \geq p_{j}^{k-1}-R D_{j} v_{j}^{k}+S D_{j}\left(v_{j}^{k-1}-v_{j}^{k}\right)+\bar{P}_{j}\left(1-v_{j}^{k-1}\right), \forall j, k \in\{2, \cdots, T\} \\
\bar{p}_{j}^{k} \leq \bar{P}_{j} v_{j}^{k+1}+S D_{j}\left(v_{j}^{k}-v_{j}^{k+1}\right), \forall j, k \in\{1, \cdots, T-1\}
\end{gathered}
$$

The minimum up and down constraints was initially expressed in [22] as a mixed integer linear expression depends concomitant with the on/off of binary variables, start-up/shutdown of generating unit's states. Further, a similar linear formulation of mixed integer based on binary variables which are presented as $v_{j}^{k}$. The novel countenance for the minimum uptime is given as follow

$$
\sum_{k=1}^{G_{j}}\left[1-v_{j}^{k}\right]=0, \forall j
$$

In (15) the $G_{j}$ is expressed mathematically. As well-defined by $G_{j}$ in (15) is subject to the preliminary position of the units which are related to constraints (14)

$$
G_{j}=\min \left\{T,\left(U T_{j}-U_{0 j}\right) V_{0 j}\right\}, \forall j
$$

To satisfy the minimum time up constraint, the (16) constraints are used for the subsequent periods during all consecutive period of size $U T_{j}$ for all possible sets.

$$
\sum_{n=k}^{k+U T_{j}-1} v_{j}^{n} \geq U T_{j}\left(v_{j}^{k}-v_{j}^{k-1}\right), \forall j, k \in\left\{G_{j}+1, \cdots, T-U T_{j}+1\right\}
$$

The Final $U T_{j-1}$ period in constraints (17) model where if unit $j$ will be started up, and continue online till the end of spam time (minimal up and downtime)

$$
\sum_{n=k}^{T}\left\{v_{j}^{k}-\left(v_{j}^{k}-v_{j}^{k-1}\right)\right\} \geq 0, \forall j, k \in\left\{G_{j}+1, \cdots, T-U T_{j}+1\right\}
$$

Similarly, the constraints for minimum downtime are formulated as follow (18)-(21) 


$$
\begin{gathered}
\sum_{k=1}^{L_{j}} v_{j}^{k}=0, \forall j \\
L_{j}=\min \left\{T,\left(D T_{j}-S_{0 j}\right)\left(1-V_{0_{j}}\right)\right\}, \forall j \\
\sum_{n=k}^{k+D T_{j}-1}\left(1-v_{j}^{n}\right) \geq D T_{j}\left(v_{j}^{k-1}-v_{j}^{k}\right), \forall j, k \in\left\{G_{j}+1, \cdots, T-U T_{j}+1\right\} \\
\sum_{n=k}^{T}\left\{1-v_{j}^{n}-\left(v_{j}^{k-1}-v_{j}^{k}\right)\right\} \geq 0, \forall j, k \in\left\{G_{j}+1, \cdots, T-U T_{j}+1\right\}
\end{gathered}
$$

In short, from the problem (1)-(21) establishes the proposed MILP-UC, that only needs binary variables of single types, namely, $v_{j}^{k}$, a contrast to the formulation of other MILP [11]. The extra binary variables definition is based on [22]. The model of [22] is also outperforms by MILP-UC by allowing for a precise model of ramping limits \& distinct contribution to the spinning reverse prerequisite.

\section{Case Studies}

\subsection{Test Data and Implementation}

In test market, a case study is carried out with hourly day ahead resolution. The examined wholesale market reflects the properties of the GB power system and includes 7 electricity producers, with their cost features, and all-out yield limits are resulting from [23] The examined retailer is assumed to serve a certain percentage of the entire population of the consumers in the wholesale market and offer the maximum allowable limit of the retail prices to its served consumers being $200 \mathrm{f} / \mathrm{MWh}$, which is imposed by the regulatory framework. The daily demand profile and the benefit characteristics of consumers are derived from [23]. Furthermore, the total generation capacity is assumed to be $10 \%$ of the renewable energy. In Figure 2 the related renewable uncertainties under normal distribution are modeled with equitable probability.

A case study with a real size is based on a ten-unit system of [20], Furthermore,

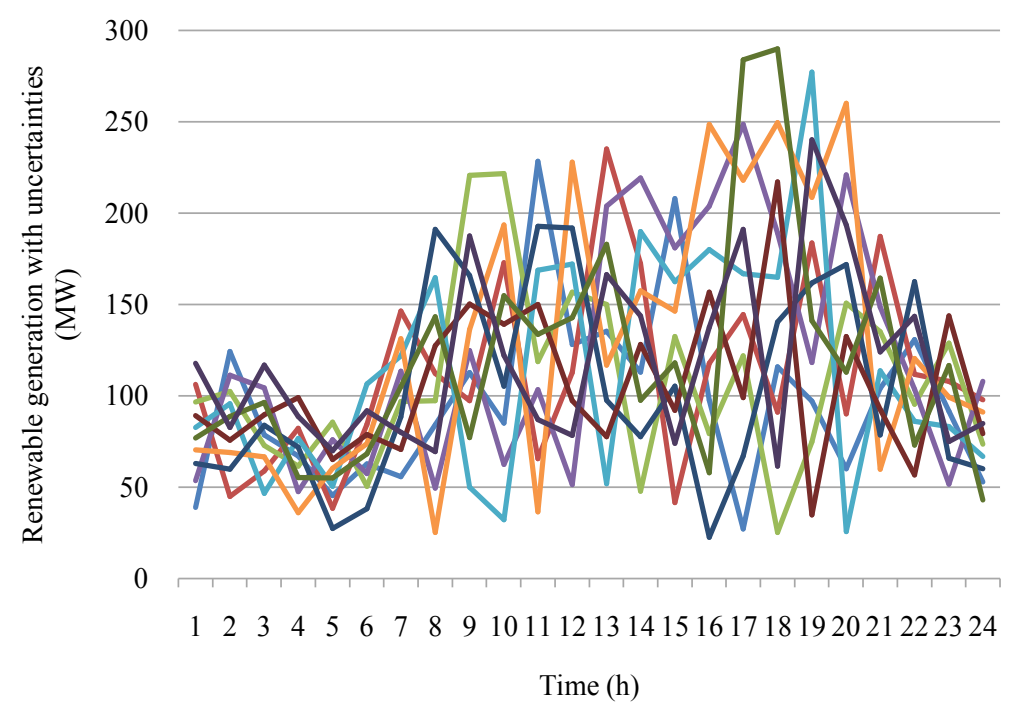

Figure 2. Power output of renewable energy with uncertainties. 
the load demand has also been multiplied by 10 accordingly. The time span is divided to meet $10 \%$ of the spinning reserve requirement each of the 24 hourly periods. The inventive ten-unit structure can be seen in the appendix [20] for quick reference. To simplify the evaluation of the fallouts, the quadratic production cost is linearized with four segments through a piecewise linear approximation. The time-dependent start-up cost has been modeled by 15 intermissions stair wise linear function. As the solution obtained from MILP-UC, a quadratic-programming-based economic dispatch is run.

The recommended model has been executed with CPLEX 9.0 [24]. The Dell Power Edge 6600 of a memory ram of $2 \mathrm{~GB}$ and with a processor of $1.60 \mathrm{GHz}$ in two numbers are used to solve the MILP with uncertainties and MINOS 5.51 [25] for the quadratic economic dispatch. In parameters can be indicated in CPLEX to find optimal solution or sub optimal solution.

Table 1 summarizes the results of three MILP centered methods which produces the similar solution when the optimal parameter of $0.25 \%$ is detailed. The computational act of the proposed method is gaged with two alike MILP formulation denoted as MILP-2 and MILP-2R respectively. The proposed methods show good results in term of total operation cost with respect to others. While comparison through reduction and computation time by 2.12, 2.67 and 3.19 respectively which shows the computational advantages of eliminating variables with respect to startup and shutdown. For the purpose of illustration, the production schedule establishes by MILP-UC is shown in Table 2. Finally, to evaluate the impact of problem size on the computational aspect of MILP-UC, MILP-2 and MILP-2R a data set of 10 units has been taken as Table 2 and applied to the ten-unit system to create 100 unit set data from the original data [20]. The computational time which is shown in Table 3 shows the satisfying solutions at an optimally tolerance of $0.25 \%$.

\subsection{Results}

Table 3 summarizes the power output which reflects the states of 10 units in 24 hours depending on the relevant unit states while in Figure 3 of Table 3 the change in data is more clearly visible. It can be seen from the table that the unit 1 is always online in 24 hours, and the output power is also up to its Start-up

Table 1. Computational dimension and results comparison.

\begin{tabular}{cccc}
\hline Name & MILP-UC & MILP-2 & MILP-2R \\
\hline Binary Variables (No's) & 2100 & 6900 & 4500 \\
Continuous Variables (No's) & 18,901 & 18,901 & 21,301 \\
Constraints (No's) & 69,189 & 74,049 & 74,049 \\
Non-zero Elements in constraints & 388,161 & 411,741 & 411,741 \\
Matrix (No's) & 113 & 314 & 399 \\
Computing Times in seconds & 564,889 & $5,611,828$ & $5,606,577$ \\
\hline
\end{tabular}


Table 2. Power output of unit-J at K period (MW).

\begin{tabular}{|c|c|c|c|c|c|c|c|c|c|c|c|c|}
\hline \multirow{2}{*}{ Unit J } & \multicolumn{12}{|c|}{ Period K } \\
\hline & 1 & 2 & 3 & 4 & 5 & 6 & 7 & 8 & 9 & 10 & 11 & 12 \\
\hline 1 & 455 & 455 & 455 & 455 & 455 & 455 & 455 & 455 & 455 & 455 & 455 & 455 \\
\hline 2 & 150 & 150 & 195 & 305 & 360 & 450 & 455 & 455 & 455 & 455 & 455 & 455 \\
\hline 3 & 20 & 65 & 130 & 130 & 130 & 130 & 130 & 130 & 130 & 130 & 130 & 130 \\
\hline 4 & 120 & 130 & 130 & 130 & 130 & 130 & 130 & 130 & 130 & 130 & 130 & 130 \\
\hline 5 & 25 & 25 & 25 & 25 & 25 & 25 & 75 & 130 & 162 & 162 & 162 & 162 \\
\hline 6 & 0 & 0 & 0 & 0 & 0 & 20 & 20 & 20 & 78 & 80 & 80 & 80 \\
\hline 7 & 0 & 0 & 0 & 0 & 0 & 0 & 0 & 0 & 0 & 25 & 63 & 85 \\
\hline 8 & 0 & 0 & 0 & 0 & 0 & 0 & 0 & 0 & 10 & 55 & 55 & 55 \\
\hline 9 & 0 & 0 & 0 & 0 & 0 & 0 & 0 & 0 & 10 & 38 & 55 & 55 \\
\hline 10 & 0 & 0 & 0 & 0 & 0 & 0 & 0 & 0 & 0 & 10 & 10 & 43 \\
\hline Sum & 770 & 825 & 935 & 1045 & 1100 & 1210 & 1265 & 1320 & 1430 & 1540 & 1595 & 1650 \\
\hline $\mathrm{D}$ & 700 & 750 & 850 & 950 & 1000 & 1100 & 1150 & 1200 & 1300 & 1400 & 1450 & 1500 \\
\hline $\mathrm{R}$ & 70 & 75 & 85 & 95 & 100 & 110 & 115 & 120 & 130 & 140 & 145 & 150 \\
\hline \multirow{2}{*}{ Unit J } & \multicolumn{12}{|c|}{ Period K } \\
\hline & 13 & 14 & 15 & 16 & 17 & 18 & 19 & 20 & 21 & 22 & 23 & 24 \\
\hline 1 & 455 & 455 & 455 & 455 & 455 & 455 & 455 & 455 & 455 & 455 & 455 & 455 \\
\hline 2 & 455 & 455 & 455 & 415 & 360 & 455 & 455 & 455 & 455 & 455 & 275 & 165 \\
\hline 3 & 130 & 130 & 130 & 130 & 130 & 130 & 130 & 130 & 130 & 130 & 130 & 130 \\
\hline 4 & 130 & 130 & 130 & 130 & 130 & 130 & 130 & 130 & 130 & 130 & 130 & 130 \\
\hline 5 & 162 & 162 & 130 & 25 & 25 & 40 & 100 & 162 & 162 & 25 & 0 & 0 \\
\hline 6 & 80 & 78 & 20 & 0 & 0 & 0 & 20 & 80 & 68 & 20 & 0 & 0 \\
\hline 7 & 25 & 0 & 0 & 0 & 0 & 0 & 0 & 0 & 0 & 0 & 0 & 0 \\
\hline 8 & 55 & 10 & 0 & 0 & 0 & 0 & 10 & 55 & 10 & 0 & 0 & 0 \\
\hline 9 & 55 & 10 & 0 & 0 & 0 & 0 & 10 & 55 & 10 & 0 & 0 & 0 \\
\hline 10 & 10 & 0 & 0 & 0 & 0 & 0 & 10 & 18 & 10 & 0 & 0 & 0 \\
\hline Sum & 1540 & 1430 & 1320 & 1155 & 1100 & 1210 & 1320 & 1540 & 1430 & 1210 & 990 & 880 \\
\hline $\mathrm{D}$ & 1400 & 1300 & 1200 & 1050 & 1000 & 1100 & 1200 & 1400 & 1300 & 1100 & 900 & 800 \\
\hline $\mathrm{R}$ & 140 & 130 & 120 & 105 & 100 & 110 & 120 & 140 & 130 & 110 & 90 & 80 \\
\hline
\end{tabular}

Table 3. Impact of problem size on computational time.

\begin{tabular}{cccc}
\hline Number of units & MILP-UC & MILP-2 & MILP-2R \\
\hline $1-10$ & 0.88 & 2.05 & 2.51 \\
$11-20$ & 3.45 & 15.12 & 14.99 \\
$21-30$ & 10.72 & 62.78 & 43.06 \\
$31-40$ & 18.48 & 75.18 & 75.04 \\
$41-50$ & 24.60 & 118.63 & 117.90 \\
$51-60$ & 36.78 & 170.15 & 160.80 \\
$61-70$ & 49.66 & 170.19 & 16.80 \\
$71-80$ & 132.83 & 215.33 & 202.92 \\
$81-90$ & 166.69 & 230.92 & 348.38 \\
$91-100$ & 172.45 & 322.95 & 408.95 \\
\hline
\end{tabular}




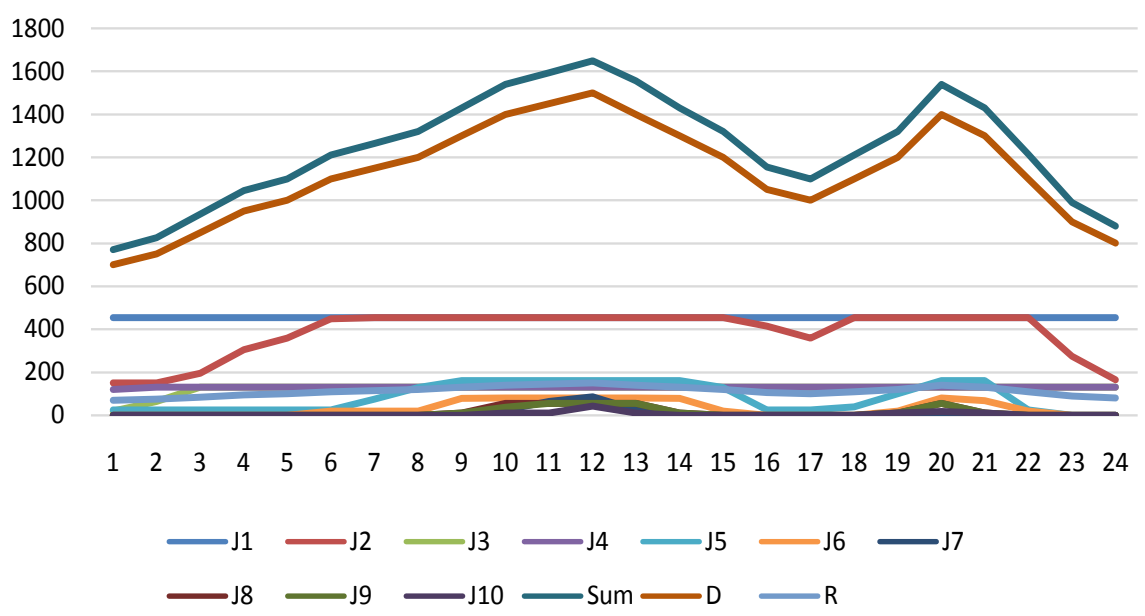

Figure 3. Power output of unit-J at K period (MW).

Ramping limit, $455 \mathrm{MW}$. However, with the operation of the system, the units start to change their states while meeting the constraints and system demand and spinning reserve requirements.

The Purple (D) line and dark green (R) line in Figure 4 represents the system demands and spinning reserve requirements respectively in 24 hours. The legend of J1 to J10 (unit1 to 10) indicate the individual unit power output in 24 hours. And it can be found, the sum of 10 units' power outputs in individual period is exactly equal to the amount of demand plus spinning reserve in the same period. Thus, it can be summarized that the system is in a good production performance (In this case, the spinning reserve is assumed to be the $10 \%$ of the demands.).

The production costs of 10 units in 24 hours are also presented in Figure 5. The production costs follow the same trend of the power output in Figure 4 for each unit and each hour period.

\subsection{Conclusion}

In this paper, the linear formulation of thermal unit commitment problem with uncertainties through a computationally efficient mixed integer is presented. The significant features of the proposed method are the single type binary numbers requirement, which precisely model the startup costs (time dependent), along with the intertemporal constraints and, especially individual contributions to spinning reserve. The problem of reduction in computation time which is normally a huge issue in unit commitment is decreased by the accessible commercial software. The recommended model has been effectively tested on a genuine situation study for wind and PV uncertainties. The numerical results show the efficient and correct computational performance. Finally, it is also applicable to the new scheduling problem arising in electricity market although the formulation has been used in traditional centralized power system for the unit commitment problem. 


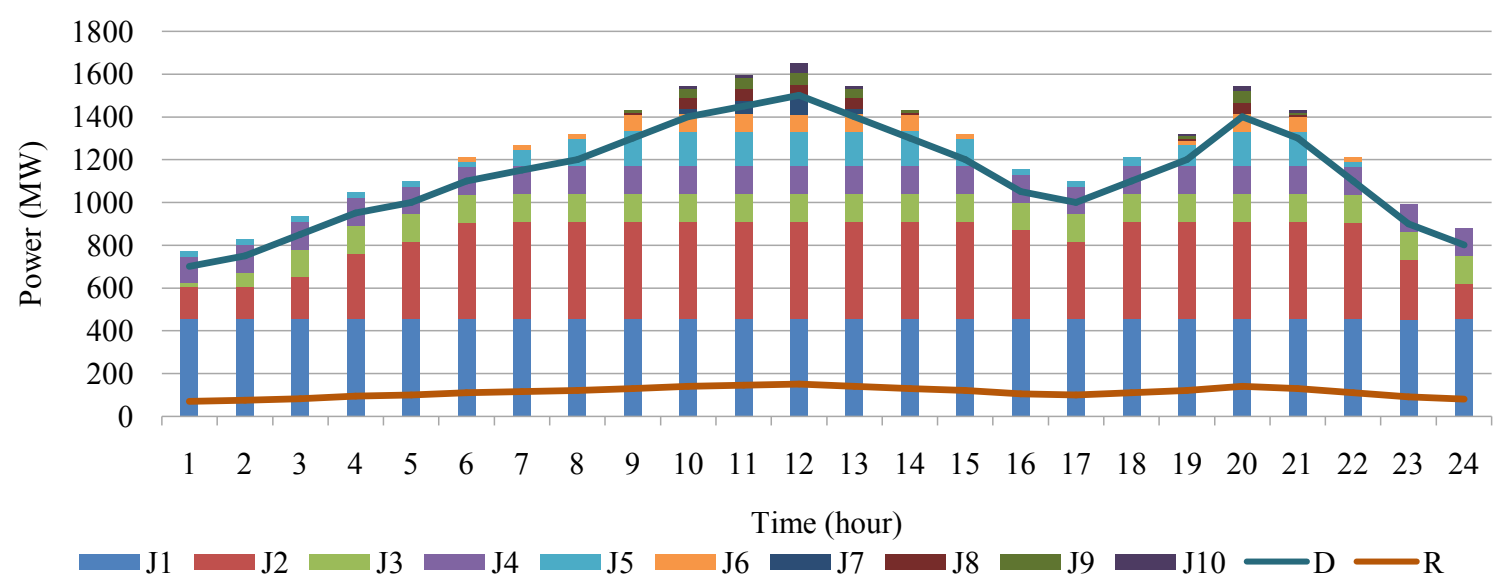

Figure 4. Hourly demand, reserve and power outputs of each generation company.

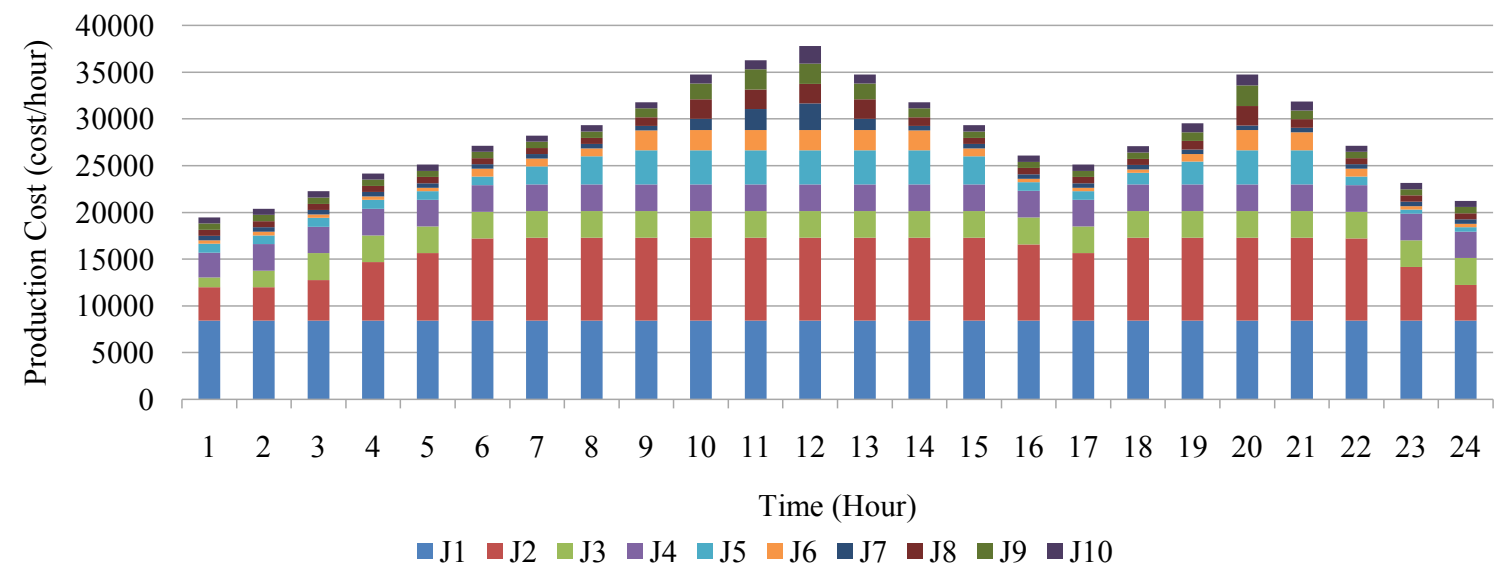

Figure 5. Hourly demand, reserve and power outputs of each generation company.

\section{Acknowledgements}

The authors would like to thank the financial support provided by the National Science Foundation under Grant 61273142, Foundation for Six Talents by Jiangsu Province (2012-DZXX-045), Priority Academic Program Development of Jiangsu Higher Education Institutions (PADP), and Technology research project of Ministry of public security of China (grant number 2015JSYJC029).

\section{References}

[1] Wood, A.J. and Wollenberg, B. (1996) Power Generation Operation and Control. 2nd Edition, In Fuel and Energy Abstracts, Vol. 37, No. 3, p. 195. Elsevier.

[2] Mohammad, S., Hatim, Y. and Li, Z.Y. (2002) Market Operations in Electric Power Systems: Forecasting, Scheduling, and Risk Management.

[3] Remedying Undue Discrimination Through Open Access Transmission Service and Standard Electricity Market Design, Notice of Proposed Rulemaking, Docket No. RM01-12-000, Federal Energy Regulatory Commission, Jul. 2002. http://www.ferc.gov/

[4] Snyder, W.L., Powell, H.D. and Rayburn, J.C. (1987) Dynamic Programming Approach to Unit Commitment. IEEE Transactions on Power Systems, 2, 339-348. 
https://doi.org/10.1109/TPWRS.1987.4335130

[5] Hobbs, W.J., Hermon, G., Warner, S. and Shelbe, G.B. (1988) An Enhanced Dynamic Programming Approach for Unit Commitment. IEEE Transactions on Power systems, 3, 1201-1205. https://doi.org/10.1109/59.14582

[6] Ouyang, Z. and Shahidehpour, S.M. (1991) An Intelligent Dynamic Programming for Unit Commitment Application. IEEE Transactions on Power Systems, 6, 1203-1209. https://doi.org/10.1109/59.119267

[7] Lee, F.N. (1988) Short-Term Thermal Unit Commitment-A New Method. IEEE Transactions on Power Systems, 3, 421-428. https://doi.org/10.1109/59.192892

[8] Li, C.-A., Johnson, R.B. and Svoboda, A.J. (1997) A New Unit Commitment Method. IEEE Transactions on Power Systems, 12, 113-119. https://doi.org/10.1109/59.574930

[9] Senjyu, T., Shimabukuro, K., Uezato, K. and Funabashi, T. (2003) A Fast Technique for Unit Commitment Problem by Extended Priority List. IEEE Transactions on Power Systems, 18, 882-888. https://doi.org/10.1109/TPWRS.2003.811000

[10] Chang, W.P. and Luo, X.J. (2008) A Solution to the Unit Commitment Using Hybrid Genetic Algorithm. In TENCON 2008-2008 IEEE Region 10 Conference, Hyderabad, 19-21 November 2008, 1-6. https://doi.org/10.1109/TENCON.2008.4766718

[11] Dillon, T.S., Edwin, K.W., Kochs, H.-D. and Taud, R.J. (1978) Integer Programming Approach to the Problem of Optimal Unit Commitment with Probabilistic Reserve Determination. IEEE Transactions on Power Apparatus and Systems, 6, 2154-2166. https://doi.org/10.1109/TPAS.1978.354719

[12] Medina, J., Quintana, V.H. and Conejo, A.J. (1999) A Clipping-Off Interior-Point Technique for Medium-Term Hydro-Thermal Coordination. IEEE Transactions on Power Systems, 14, 266-273. https://doi.org/10.1109/59.744542

[13] Carrión, M. and Arroyo, J.M. (2006) A Computationally Efficient Mixed-Integer Linear Formulation for the Thermal Unit Commitment Problem. IEEE Transactions on Power Systems, 21, 1371-1378. https://doi.org/10.1109/TPWRS.2006.876672

[14] Xie, J., Zhong, J., Li, Z. and Gan, D. (2011) Environmental-Economic Unit Commitment Using Mixed-Integer Linear Programming. International Transactions on Electrical Energy Systems, 21, 772-786. https://doi.org/10.1002/etep.476

[15] Guerrero-Mestre, V. and Contreras, J. (2016) Stochastic Unit Commitment Considering Start-Up and Shut-Down Trajectories. IEEE Power and Energy Society General Meeting (PESGM), Boston, 17-21 July 2016, 1-5.

https://doi.org/10.1109/PESGM.2016.7741655

[16] Zhuang, F. and Galiana, F.D. (1990) Unit Commitment by Simulated Annealing. IEEE Transactions on Power Systems, 5, 311-318. https://doi.org/10.1109/59.49122

[17] Mantawy, A.H., Youssef, L., Abdel-Magid, Y.L. and Selim, S.Z. (1998) A Simulated Annealing Algorithm for Unit Commitment. IEEE Transactions on Power Systems, 13, 197-204. https://doi.org/10.1109/59.651636

[18] Merlin, A. and Sandrin, P. (1983) A New Method for Unit Commitment at Electricite De France. IEEE Transactions on Power Apparatus and Systems, 5, 1218-1225. https://doi.org/10.1109/TPAS.1983.318063

[19] Ongsakul, W. and Petcharaks, N. (2004) Unit Commitment by Enhanced Adaptive Lagrangian Relaxation. IEEE Transactions on Power Systems, 19, 620-628. 
https://doi.org/10.1109/TPWRS.2003.820707

[20] Kazarlis, S.A., Bakirtzis, A.G. and Petridis, V. (1996) A Genetic Algorithm Solution to the Unit Commitment Problem. IEEE Transactions on Power Systems, 11, 83-92. https://doi.org/10.1109/59.485989

[21] Nowak, M.P. and Römisch, W. (2000) Stochastic Lagrangian Relaxation Applied to Power Scheduling in a Hydro-Thermal System under Uncertainty. Annals of Operations Research, 100, 251-272. https://doi.org/10.1023/A:1019248506301

[22] Arroyo, J.M. and Conejo, A.J. (2000) Optimal Response of a Thermal Unit to an Electricity Spot Market. IEEE Transactions on Power Systems, 15, 1098-1104. https://doi.org/10.1109/59.871739

[23] Svoboda, A.J., Tseng, C.-L., Li, C.-A. and Johnson, R.B. (1997) Short-Term Resource Scheduling with Ramp Constraints [Power Generation Scheduling]. IEEE Transactions on Power Systems, 12, 77-83. https://doi.org/10.1109/59.574926

[24] CPLEX, ILOG (2006). http://www.ilog.com/products/cplex

[25] Saunders, M.A., Murray, W. and Gill, P.E. (2002) MINOS: A Solver for Large-Scale Nonlinear Optimization Problems. 


\section{Appendix}

In Table A1 the data of Ten-unit system is provided [20].

Table A1. The system data.

\begin{tabular}{|c|c|c|c|c|c|c|}
\hline Unit J & $\bar{p}_{j} \quad(\mathrm{MW})$ & $\underline{P}_{j}$ & $(\mathrm{MW})$ & UT (h) & DT (h) & $V_{0}(\mathrm{~h})$ \\
\hline 1 & 455 & & 150 & 8 & 8 & 8 \\
\hline 2 & 455 & & 150 & 8 & 8 & 8 \\
\hline 3 & 130 & & 20 & 5 & 5 & -5 \\
\hline 4 & 130 & & 20 & 5 & 5 & -5 \\
\hline 5 & 162 & & 25 & 6 & 6 & -6 \\
\hline 6 & 80 & & 20 & 3 & 3 & -3 \\
\hline 7 & 55 & & 25 & 3 & 3 & -3 \\
\hline 8 & 55 & & 10 & 1 & 1 & -1 \\
\hline 9 & 55 & & 10 & 1 & 1 & -1 \\
\hline 10 & 55 & & 10 & 1 & 1 & -1 \\
\hline Unit J & & $\mathrm{a}(\mathcal{E} / \mathrm{h})$ & & $\mathrm{b}(\mathcal{E} / \mathrm{MWh})$ & & $\mathrm{C}(\mathfrak{E} / \mathrm{MW} 2 \mathrm{~h})$ \\
\hline 1 & & 1000 & & 16.19 & & 0.00048 \\
\hline 2 & & 970 & & 17.26 & & 0.00031 \\
\hline 3 & & 700 & & 16.60 & & 0.00200 \\
\hline 4 & & 680 & & 16.65 & & 0.00211 \\
\hline 5 & & 450 & & 19.70 & & 0.00398 \\
\hline 6 & & 370 & & 22.26 & & 0.00712 \\
\hline 7 & & 480 & & 27.74 & & 0.00079 \\
\hline 8 & & 660 & & 25.92 & & 0.00413 \\
\hline 9 & & 665 & & 27.27 & & 0.00222 \\
\hline 10 & & 670 & & 27.79 & & 0.00173 \\
\hline
\end{tabular}




\section{Nomenclature}

\section{Indices}

$j \in J \quad$ Set of generation units

$k \in K \quad$ Set of periods

\section{Parameters}

$\underline{P}_{j} \quad$ The Minimum generation limit of generation unit- $j$ (MW)

$\bar{P}_{j} \quad$ The Maximum generation limit of generation unit $j(\mathrm{MW})$

$U T_{j} \quad$ The Minimum start-up time of generation unit- $j(\mathrm{~s})$

$D T_{j} \quad$ The Minimum shunt down time of generation unit- $j(\mathrm{~s})$

$U_{0 j} \quad$ The time of Initial start-up to generation unit $j$ (s)

$S_{0 j} \quad$ The time of Initial shunt-down to generation unit- $j(\mathrm{~s})$

$V_{0 j} \quad$ Initial commitment state of generation unit- $j$ (online: 1 , offline: 0 )

$a_{j} \quad$ The coefficient of Fixed quadratic production cost function to generation unit $j(\varepsilon / \mathrm{h})$

$b_{j} \quad$ The Linear coefficient for the quadratic production cost function of generation unit- $j(\varepsilon / \mathrm{MWh})$

$c_{j} \quad$ Quadratic coefficient for the quadratic production cost function of generation unit $-j\left(£ / \mathrm{MWh}^{\wedge} 2\right)$

$A_{j}^{k} \quad$ Cost of generation unit $j$ at $k(\mathfrak{E})$ period

$B_{j}^{k} \quad$ The Cost of generation unit $j$ at $k(\mathfrak{E})$ period

$C U_{j}^{t} \quad$ The cost of Maximum start-up for generation unit- $j$ at period $k$ $(E / \mathrm{MWh})$

$C D_{j} \quad$ The cost of Maximum shut down for generation unit- $j$ at period $k$ (E/MWh)

$S U_{j} \quad$ Start-up ramping of generation unit- $j(\mathrm{MW})$

$S D_{j} \quad$ Shunt down ramping of generation unit $-j(\mathrm{MW})$

$D^{K} \quad$ Demand at period $K(\mathrm{MW})$

$R^{K} \quad$ Reverse at period $K(\mathrm{MW})$

\section{Variables}

$v_{j}^{k} \quad$ On-Off state of generation unit- $j$ at period $t[0 / 1]$

$p_{j}^{k} \quad$ Generation output of generation unit- $j$ at period $t(\mathrm{MW})$

$c u_{j}^{t} \quad$ Start-up cost of generation unit- $j$ at period $k(E / \mathrm{MWh})$

$c d_{j}^{k} \quad$ The cost of Shunt down for generation unit $j$ at the $k$ period ( $\left.E / \mathrm{MWh}\right)$ 